\title{
Systematic Review of Antimicrobial Drug Prescribing in Hospitals
}

\author{
Peter Davey, ${ }^{*} \dagger$ Erwin Brown, $\ddagger$ Lynda Fenelon,§ Roger Finch, $\Uparrow \#$ lan Gould, ${ }^{* *}$ Alison Holmes, $\dagger \dagger$ \\ Craig Ramsay, $\ddagger$ Eric Taylor,§§ Phil Wiffen,
}

Prudent prescribing of antimicrobial drugs to hospital inpatients may reduce incidences of antimicrobial drug resistance and healthcare-associated infection. We reviewed the literature from January 1980 to November 2003 to identify rigorous evaluations of interventions to improve hospital prescribing of antimicrobial drugs. We identified 66 studies with interpretable data, of which 16 reported 20 microbiologic outcomes: gram-negative resistant bacteria, 10 studies; Clostridium difficile-associated diarrhea, 5 studies; vancomycin-resistant enterococci, 3 studies; and methicillin-resistant Staphylococcus aureus, 2 studies. Four studies provided strong evidence that the intervention changed microbial outcomes with low risk for alternative explanations, 8 studies provided less convincing evidence, and 4 studies provided no evidence. The strongest and most consistent evidence was for $C$. difficile-associated diarrhea, but we were able to analyze only the immediate impact of interventions because of nonstandardized durations of follow-up. The ability to compare results of studies could be substantially improved by standardizing methods and reporting.

$\mathrm{D}$ espite strenuous efforts to control antimicrobial drug use and promote optimal prescribing, practitioners continue to prescribe excessively; it is estimated that up to $50 \%$ of antimicrobial drug use in hospitals is inappropriate (1-3). Antimicrobial drug resistance is largely a consequence of the selective pressures of antimicrobial drug use.

\footnotetext{
*University of Dundee Medical School, Dundee, United Kingdom; †Ninewells Hospital, Dundee, United Kingdom; ‡Frenchay Hospital, Bristol, United Kingdom; §St Vincent's University Hospital, Dublin, Ireland; đNottingham City Hospital, Nottingham, United Kingdom; \#University of Nottingham, Nottingham, United Kingdom; **Aberdeen Royal Infirmary, Aberdeen, United Kingdom; ††Hammersmith Hospital, London, United Kingdom; łłUniversity of Aberdeen Health Services Research Unit, Aberdeen, United Kingdom; §§Inverclyde Royal Hospital, Greenock, United Kingdom; ףा United Kingdom Cochrane Centre, Oxford, United Kingdom; \#\#Leeds General Infirmary, Leeds, United Kingdom; and ${ }^{* *}$ University of Leeds, Leeds, United Kingdom
}

Reducing these pressures by the judicious administration of these drugs should facilitate a return of susceptible bacteria or, at least, prevent or slow the pace of the emergence of drug-resistant strains $(4,5)$. Furthermore, Clostridium difficile-associated diarrhea (CDAD) is a hospitalacquired infection associated with use of antimicrobial drugs (6-8) and reducing the incidences of CDAD is an additional potential benefit of improving hospital antimicrobial drug prescribing.

Implementing and monitoring interventions to optimize prescribing of antimicrobial drugs place a burden on hospital resources and their efficacies need to be confirmed (9). We have conducted a systematic review of interventions to improve antimicrobial drug-prescribing practices for hospital inpatients using the methods of the Cochrane Effective Practice and Organization of Care Group to assess validity (10). In this study, our primary objective was to evaluate the impact of interventions on reducing the incidence of colonization with or infection caused by antimicrobial drug-resistant pathogens or CDAD. In addition to the usual threats to the validity of interventions to change health care, infection control interventions are particularly prone to regression to the mean (11). This refers to the natural tendency of extreme observations to return towards the average (mean) over time. An epidemic or outbreak is a sequence of unusually large number of cases of infection, so that the natural history of an epidemic is to increase, peak, and then decrease. Consequently, regression to the mean is always a threat to the validity of evaluations of unplanned interventions that are initiated in response to an outbreak.

\section{Methods}

The full protocol is available in the Cochrane Library (10). We searched Medline, EMBASE, the Cochrane database, and the Effective Practice and Organisation of Care specialized register for studies from January 1, 1980, to November 30, 2003, relating to prescribing of antimicrobial 
drugs to hospital inpatients. Additional studies were obtained from the bibliographies of retrieved articles, the Scientific Citation Index, and personal files. We requested additional data from the authors when necessary. There were no language limitations for the literature review. We included all randomized and controlled clinical trials (RCT/CCT, designs where allocation to the intervention is determined either by an explicit random process [RCT] or by a nonrandom process such as date of birth or case note number) before and after studies (a design with contemporaneous data collection before and after the intervention and an appropriate control site or activity) and interrupted time series (ITS, a clearly defined point in time when the intervention occurred and at least 3 data points before and 3 after the intervention). Data about microbiologic outcomes were considered reliable if they met the same criteria. For example, if a paper included prescribing data that met the criteria for an ITS but provided only mean data about microbiologic outcomes before and after the intervention, then the microbiologic data were not considered reliable. Two reviewers independently extracted data and assessed the quality of each study with the standardized criteria.

\section{Statistical Considerations}

Many statistical methods can be used to analyze ITS designs (e.g., ARIMA modeling or time series regression). However, the design is often analyzed inappropriately, which makes interpretation of individual studies difficult (12). Methods of analyzing ITS data were examined critically (12). The preferred method for short time series is segmented time series regression analysis, which is a statistical comparison of time trends before and after the intervention to identify either an immediate change in the level of the regression line or a sustained change in the slope of the line $(12,13)$. In this report, we have distinguished 2 intervention effects: immediate (a sudden change in the level of the regression line at the point of intervention) and sustained (a sustained change in the slope of the regression line from the start of the intervention phase). If the original report did not include an appropriate analysis, data were reanalyzed by using segmented time series regression.

The following model was specified: $Y_{\mathrm{t}}=B_{0}+B_{1} \times$ preslope $+B_{2} \times$ postslope $+B_{3} \times$ intervention $+e_{\mathrm{t}}$, where $Y_{\mathrm{t}}$ is the outcome (e.g., CDAD incidence) in month $t$, preslope is a continuous variable indicating time from the start of the study up to the last point in the preintervention phase and coded constant thereafter, postslope is coded 0 to and including the first point postintervention and coded sequentially from 1 thereafter, and intervention is coded 0 for preintervention time points and 1 for postintervention time points. In this model, $B_{1}$ estimates the slope of the preintervention data, $B_{2}$ estimates the slope of the postin- tervention data, and $B_{3}$ estimates the change in level of outcome as the difference between the estimated first point postintervention and the extrapolated first point postintervention if the preintervention line was continued into the postintervention phase. The difference in slope is calculated by $B_{2}-B_{1}$. The error term $e_{\mathrm{t}}$ was assumed to be firstorder autoregressive. Confidence intervals (95\%) were calculated for all effect measures.

Formal metaanalysis of results was not attempted given the differences in context, setting, and type of outcomes. However, to gain an overall summary picture of the heterogeneity of effect sizes we standardized all measures so that they were all on the same scale. To do this, we divided the change in level and the change in slope by the preintervention standard deviation (SD) in each study. The resulting metric has no unit, it is known in standard metaanalysis as the standardized mean difference. Standardized effect sizes of 2 to 3 SD were considered large, whereas an effect size $<0.5$ SD was considered of questionable clinical significance even if statistically significant (14). To visually display the heterogeneity of the standardized effect sizes, graphic plots of level effects versus slope effects for each study (with associated 95\% confidence intervals) were generated.

\section{Other Criteria for Assessing Evidence}

The statistical analysis assessed how likely it was that study results could simply have happened by chance, and the Cochrane quality criteria assessed common threats to the validity of interventions to change practice or organization of care. To assess other threats to the validity of infection control interventions, we used the format for reporting the results of included studies recommended by guidelines derived from a recent systematic review of isolation measures to control methicillin-resistant Staphylococcus aureus (MRSA) (15). We required studies to provide reliable data about the effect of interventions on both microbial and drug outcomes with clear case definition, description of infection control measures, and other variables such as bed occupancy or staffing levels that could provide plausible alternative explanations for changes in microbial outcomes. We have provided a summary of detailed information from the included studies (online Appendix Table, available from http://www.cdc. gov/ncidod/EID/vol12no02/05-0145.htm\#apptable). Additional information is available from the British Society for Antimicrobial Chemotherapy (www.bsac. org.uk). We classified case definitions into colonization, infection or clinical isolates, or a combination of $\geq 2$ with the following definitions.

Colonization was defined as a microorganism, usually detected by screening, at a host site (normally nonsterile, although the urine of a catheterized patient may be an 
exception) without causing systemic signs of infection or a specific immune response. Colonization by case note review was established by excluding infection diagnosed according to criteria adopted by the authors or defined by appropriate bodies, e.g., the Centers for Disease Control and Prevention criteria for diagnosing nosocomial infections. Infection was established by case note review according to criteria adopted by the authors or defined by appropriate bodies or by recording specific symptoms and/or signs, such as diarrhea in patients with CDAD. Clinical isolates were defined as the recovery of a microorganism after culture of a clinical (not screening) specimen without specifying whether it represents colonization or infection.

\section{Results}

We identified 66 intervention studies to improve prescribing of antimicrobial drugs to hospital inpatients that met our inclusion criteria (16) and excluded 243 studies that were uncontrolled before and after studies $(n=164)$ or inadequate ITS studies $(n=79)$. Of the 66 studies, 16 reported reliable data about 20 microbiologic outcomes: gram-negative resistant bacteria (GNRB), 10 studies; CDAD, 5 studies; vancomycin-resistant enterococci (VRE), 3 studies; and MRSA, 2 studies (online Appendix Table). The setting for the intervention was the entire hospital in 8 studies (17-24), a single service in 2 studies $(25,26)$, and a unit or ward in 6 studies $(27-32)$. One intervention was educational with advice about changes in antimicrobial drugs (17); the other 15 interventions were restrictive (online Appendix Table). Two studies were RCTs $(31,32)$ and 1 study was a CCT $(30)$; the remaining 13 studies used an ITS design.

\section{Statistical Validity}

All 3 clinical trials reported appropriate statistical analysis (30-32), whereas only 2 of the 13 ITS studies reported appropriate statistical analysis $(17,27)$. Of the remaining 11 ITS studies, 5 did not report statistical analysis; 6 reported inappropriate statistical analysis by using tests such as $\chi^{2}$ or $t$ tests that assume independence between observations and do not account for time trends. Data from these 11 studies were reanalyzed.

\section{Effectiveness of Interventions}

Overall, 4 studies provided strong evidence of control of the microbial outcome by change in prescribing $(17,27,30,31)$. All of these studies provided reliable data about antimicrobial drug prescribing, with significant changes in both microbial and drug outcomes after planned interventions. In addition, 2 studies provided further protection against regression to the mean by using a crossover design $(27,30)$. Three of these studies have rigorous case definitions based on prospective screening cultures plus full description of infection control measures.

Eight studies provided less convincing evidence. Two studies showed significant changes in prescribing that were associated with nonsignficant changes in CDAD $(20,26)$. An additional 6 studies reported statistically significant improvement in microbial outcome but without reliable data about the effect of the intervention on prescribing $(18,19,23,24,28,29)$. The importance of this omission is confirmed by the 6 studies that included reliable data about prescribing because all showed that there was some prescription of restricted drugs during the intervention phase $(17,20,26,27,30,31)$

Four studies had negative results $(21,22,25,32)$. One study provided strong evidence of failure to control microbial outcomes despite a successful change in prescribing (32). One study reported an intervention that failed to change use of vancomycin (22). The remaining 2 studies showed no change in microbial outcome but did not provide reliable data about the effect of the intervention on prescribing $(21,25)$.

\section{CDAD}

The most consistent evidence was for the 5 interventions designed to reduce the incidence of CDAD. Four were implemented throughout the hospital $(17,18,20,24)$ and 1 was implemented in the elderly care service (26); all 5 targeted prescribing of cephalosporin or clindamycin. All of the interventions were associated with a change in the expected direction (Figure part A), which was a change in the incidence of CDAD in the same direction to a change in use of cephalosporin or clindamycin. For 1 intervention, the expected direction was an increase in CDAD incidence after the introduction of ceftriaxone (20); for all other interventions a decrease in CDAD incidence was expected to accompany a decrease in use of cephalosporin or clindamycin. These 5 studies reported 7 interventions. The immediate effect after 6 of the 7 interventions was at least 0.5 SDs; 5 of these 7 immediate effects were statistically significant (Figure part A). Sustained changes after the intervention were more modest, but all were in the expected directions and 4 of 7 were statistically significant (Figure part A.). The 5 CDAD studies had results expressed in different units: cases per month $(24,26)$; cases per quarter $(18,20)$; or cases per 1,000 admissions per year (17). Consequently, we were only able to compare effect sizes in numbers of CDAD cases per quarter by recalculating results from 2 studies $(24,26)$. The antimicrobial drug intervention was associated with a mean immediate reduction of 15.0 CDAD cases per quarter (range 6-26) and a median sustained reduction of 3.2 CDAD cases per quarter (range 1-6). 


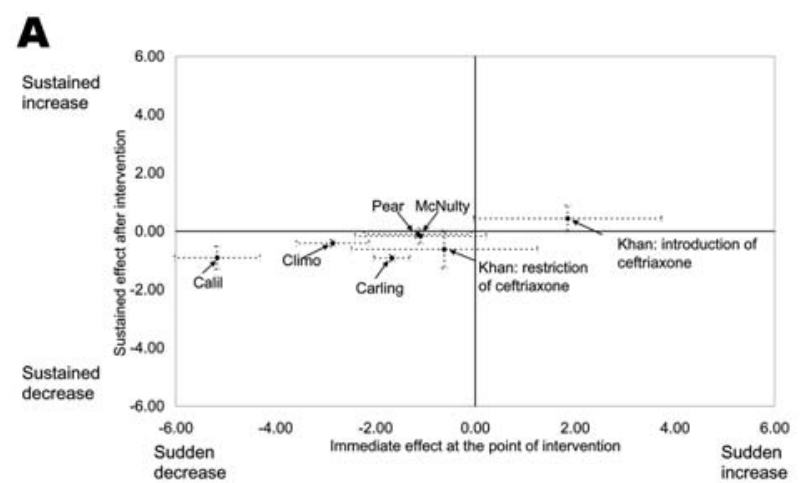

B

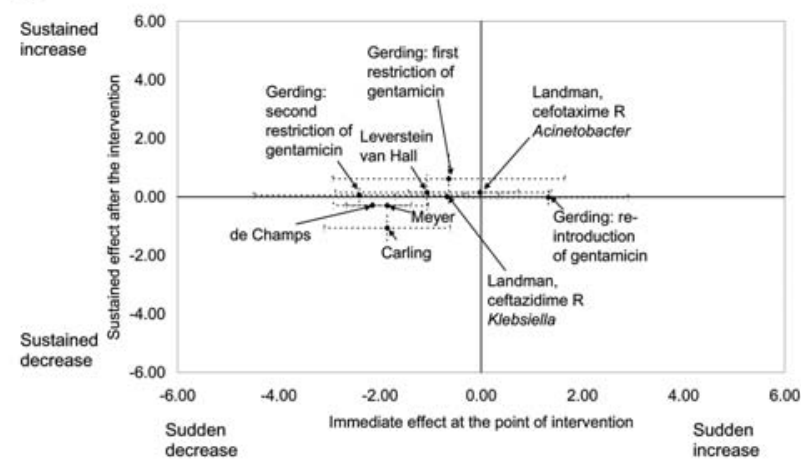

Figure. A) Standardized immediate and sustained effects for Clostridium difficile-associated diarrhea. B) Standardized immediate and sustained effects for resistant gram-negative bacteria.

\section{Resistant Gram-negative Bacteria}

The results of the 10 interventions designed to reduce the incidences of GNRB were less consistent. Three were implemented throughout the hospital $(19,21,23), 1$ was implemented in the neurology and neurosurgery service (25), and 5 were implemented in a single intensive care unit (ICU), which included 4 with pediatric patients $(28-30,32)$ and 1 with adult patients (31). One intervention was designed to reduce the duration of treatment with any antimicrobial drug for ICU patients at low risk for pneumonia; this was associated with a significant reduction in the incidence of colonization by any GNRB and exposure to antimicrobial drugs (31). The remaining 9 interventions involved changes in antimicrobial drug treatment, mainly aminoglycosides or cephalosporins. One RCT provided no evidence that antimicrobial drug cycling reduced the incidence of GNRB in a neonatal ICU (32). The 8 ITS studies reported 9 outcomes (Figure part B). The expected direction of effect from a change in aminoglycoside or cephalosporin prescribing was usually a reduction in GNRB. For 1 intervention, the expected direction of effect was an increase in the incidence of GNRB after gentamicin was reintroduced (19). The expected direction for all 9 outcomes changed, but the effect size was $<0.5$ SD in 2 studies and not statistically significant in 5 studies (Figure part
B). In 3 studies the changes in slope were in the expected direction and in 1 the changes were both statistically significant and $>0.5 \mathrm{SD}$, which is likely clinically important. Unlike with CDAD data, effects cannot be expressed in a common unit. Some studies measured colonization and others examined infection. Units of measurement were also variable (e.g., number of isolates, percentage of isolates, number of cases, and number of cases per time period).

\section{Gram-positive Bacteria}

Data for gram-positive bacteria were very limited. One study provided strong evidence that restricting ceftazidime in a hematology unit was associated with significant reduction in risk for colonization by VRE (27). However, reduction of cephalosporin use in a hospital was not associated with any change in the prevalence of VRE isolates (17). A third study targeted at VRE showed that implementation of a vancomycin order form had no significant impact on vancomycin prescribing, with a trend in the unintended direction (22). Two studies report effects on MRSA prevalence $(17,21)$. Our segmented regression analysis showed no significant change in response to a reduction in use of third-generation cephalosporins (online Appendix Table), although 1 of the reports claimed that a change did occur (21).

\section{Discussion}

Our primary conclusion is that 4 of the 16 studies provided strong evidence that changes in prescribing antimicrobial drugs to hospital inpatients can improve microbial outcomes $(17,27,30,31)$. Eight of the remaining studies provided some evidence that antimicrobial drug-prescribing interventions can improve microbial outcomes, but flaws in their design indicated that there were plausible alternative explanations for the results (18-20,23, $24,26,28,29)$. The remaining 4 studies were unequivocally negative $(21,22,25,32)$.

Estimation of overall effect size was only possible for reduction in $\mathrm{CDAD}$, where the evidence suggested that restriction of clindamycin or third-generation cephalosporins resulted in an immediate reduction in prevalence by 15 cases per quarter, with an additional sustained reduction by 3 cases per quarter. Prevalence is usually adjusted for clinical activity, e.g., cases per 1,000 admissions per quarter (7), but only 1 study provided this information (17). Furthermore, potentially important differences in the case definitions of CDAD occurred between the studies in our review.

Finding valid studies required painstaking analysis of a huge volume of literature, most of which is fundamentally flawed (16). The included studies could be dramatically improved by following guidelines for standardized reporting (15). In particular, the unequal duration of postinterven- 
tion phases made it difficult to reliably compare the sustained effects of interventions, these being the most important outcome measures. The short and unequal duration of preintervention phases provides limited information about underlying preintervention trends. To understand how much of a change in prescribing is required to change outcome, the intervention must be independent of other control measures and be accompanied by reliable data about both prescribing and microbial outcomes.

Only 1 of the interventions was designed to reduce overall exposure to antimicrobial drugs (31). All of the other studies targeted the choice of antimicrobial drug (e.g., by restricting access to third-generation cephalosporins in favor of drugs recommended by the hospital antimicrobial drug policy) but did not aim to shorten the duration of treatment. This intervention (31) shortened the duration of antimicrobial drug treatment for ICU patients at low risk for ventilator-associated pneumonia. This study was conducted in an ICU with adult patients. However, the same principle of using clinical scores to identify low-risk patients, in whom antimicrobial drug therapy could be stopped, has been developed in other clinical settings (33-35), and the impact on microbiologic outcomes should be investigated.

None of the studies provided evidence for cost-effectiveness or clinical outcome. The study designs likely did not have sufficient power to measure these outcomes. Few studies provided data about multiple microbiologic species and 1 of these endpoints (incidence of cefotaxime-resistant Acinetobacter spp.) was opposite to that which was expected (21). Future studies should provide more data about cost and clinical outcomes. Notably, evidence is needed to show that interventions do not have adverse outcomes.

The potential for the success of antimicrobial drug interventions likely varies by organism $(36,37)$. Antimicrobial drugs are likely to play a large role in the selection of enterobacteria expressing extended-spectrum $\beta$-lactamases, a minimal role in the selection and transmission of MRSA, and an intermediate role in VRE. However, the available evidence is not sufficient to investigate these hypotheses.

\section{Implications for Practice}

The evidence supports the theory that limiting the use of specific antimicrobial drugs will reduce the prevalences of resistant gram-negative bacteria and CDAD. For grampositive bacteria, there is a lack of evidence rather than evidence of no effect. Hospitals would like to know how much they should limit their antimicrobial drug prescriptions and what is the minimum that will show a real effect. Unfortunately, the available evidence is too limited to provide definitive answers to these issues. Thus, hospitals must estimate the effect of their own interventions. The good news is that the data required for ITS analysis of the incidences of drug-resistant bacteria or CDAD should be readily available in most hospitals. Healthcare providers need to invest in data analysis so that evaluation of antimicrobial drug control in hospitals becomes a routine measure of the quality of care rather than a research project.

Standardized reporting of outbreaks and interventions to control the incidence of antimicrobial drug resistance or hospital-acquired infection would greatly enhance the ability to combine results from hospitals in metaanalyses. Key issues include full description of other infection control measures, consistent and reproducible case definitions, the length of preintervention and postintervention phases, and the intervals between data points (15). Ideally, data should be made available in a way that allows reanalysis and, where appropriate, metaanalysis. Metaanalysis of single hospital studies is no substitute for good multicenter studies, but it could be used to provide some evidence of reproducibility and thus to prioritize targets for definitive trials.

\section{Priorities for Research}

The research agenda needs to move to multicenter studies with randomized allocation to interventions. This will provide better evidence of external validity as well as the power to measure cost-effectiveness and exclude important unintended adverse clinical outcomes. Development and pilot testing of the effectiveness of clinical decisions for reducing unnecessary exposure to antimicrobial drugs should be a priority for research in hospitals.

This study was supported by a working party grant from the British Society for Antimicrobial Chemotherapy and the Hospital Infection Society.

Dr Davey is honorary consultant in infectious diseases at the Acute Services Division of National Health Service Tayside and director of the Health Informatics Centre, a multidisciplinary group developing innovative methods for linkage and application of information from health records. His main research interests are epidemiology of antimicrobial drug prescribing or resistance and quality improvement of prescribing practices.

\section{References}

1. Behar P, Wagner MB, Freitas I, Auler A, Selistre L, Fossatti L, et al. Assessing the antimicrobial prescription request process in a teaching hospital in Brazil: regulations and training. Braz J Infect Dis. 2000;4:76-85.

2. European Union Conference. The Copenhagen Recommendations. Report from the invitational EU conference on the microbial threat. Ministry of Health, Ministry of Food, Agriculture and Fisheries, Denmark. 1998 [cited 2005 Nov 4]. Available from http://www. im.dk/publikationer/micro98/index.htm

3. Lawton RM, Fridkin SK, Gaynes RP, McGowan JE. Practices to improve antimicrobial use at 47 US hospitals: the status of the 1997 SHEA/IDSA position paper recommendations. Infect Control Hosp Epidemiol. 2000;21:256-9. 
4. Goldmann DA, Weinstein RA, Wenzel RP, Tablan OC, Duma RJ, Gaynes RP, et al. Strategies to prevent and control the emergence and spread of antimicrobial-resistant microorganisms in hospitals. A challenge to hospital leadership. JAMA. 1996;275:234-40.

5. Shlaes DM, Gerding DN, John JF, Craig WM, Bornstein DL, Duncan RA, et al. Society for Healthcare Epidemiology of America and Infectious Diseases Society of America Joint Committee on the Prevention of Antimicrobial Resistance: guidelines for the prevention of antimicrobial resistance in hospitals. Clin Infect Dis. 1997;25:584-99.

6. Bartlett JG. Antibiotic-associated diarrhoea. Clin Infect Dis. 1992;15:573-81.

7. Wilcox MH, Smyth ETM. Incidence and impact of Clostridium difficile infection in the UK, 1993-1996. J Hosp Infect. 1998;39:181-7.

8. Wistrom J, Norrby SR, Myhre EB, Eriksson S, Granstrom G, Lagergren L, et al. Frequency of antibiotic-associated diarrhoea in 2,462 antibiotic-treated hospitalized patients: a prospective study. J Antimicrob Chemother. 2001;47:43-50.

9. McGowan JE. Success, failures and costs of implementing standards in the USA: lessons for infection control. J Hosp Infect. 1995;30(Suppl):76-87.

10. Davey P, Brown E, Hartman G, Ramsay C, Wiffen P, Fenelon L, et al. Interventions to improve antibiotic prescribing practices for hospital inpatients. The Cochrane Database of Systematic Reviews. 2005 [cited 2005 Nov 4]. Available from http://www.mrw.interscience. wiley.com/cochrane/clsysrev/articles/CD003543/frame.html

11. Cooper BS, Stone SP, Kibbler CC, Cookson BD, Roberts JA, Medley $\mathrm{GF}$, et al. Isolation measures in the hospital management of methicillin resistant Staphylococcus aureus (MRSA): systematic review of the literature. BMJ. 2004;329:533.

12. Ramsay CR, Matowe L, Grilli R, Grimshaw JM, Thomas RE. Interrupted time series designs in health technology assessment: lessons from two systematic reviews of behavior change strategies. Int J Technol Assess Health Care. 2003;19:613-23.

13. Wagner AK, Soumerai SB, Zhang F, Ross-Degnan D. Segmented regression analysis of interrupted time series studies in medication use research. J Clin Pharm Ther. 2002;27:299-309.

14. Cohen J. Statistical power analysis for the behavioral sciences. London: Academic Press; 1977.

15. Cooper BS, Stone SP, Kibbler CC, Cookson BD, Roberts JA, Medley GF, et al. Systematic review of isolation policies in the hospital management of methicillin-resistant Staphylococcus aureus: a review of the literature with epidemiological and economic modelling. Health Technol Assess. 2003;7:1-194.

16. Ramsay C, Brown E, Hartman G, Davey P. Room for improvement: a systematic review of the quality of evaluations of interventions to improve hospital antibiotic prescribing. J Antimicrob Chemother. 2003;52:764-71.

17. Carling P, Fung T, Killion A, Terrin N, Barza M. Favorable impact of a multidisciplinary antibiotic management program conducted during 7 years. Infect Control Hosp Epidemiol. 2003;24:699-706.

18. Climo MW, Israel DS, Wong ES, Williams D, Coudron P, Markowitz SM. Hospital-wide restriction of clindamycin: effect on the incidence of Clostridium difficile-associated diarrhea and cost. Ann Intern Med. 1998;128:989-95.

19. Gerding DN, Larson TA. Aminoglycoside resistance in gram-negative bacilli during increased amikacin use. Comparison of experience in 14 United States hospitals with experience in the Minneapolis Veterans Administration Medical Center. Am J Med. 1985;79:1-7.

20. Khan R, Cheesbrough J. Impact of changes in antibiotic policy on Clostridium difficile-associated diarrhoea (CDAD) over a five-year period in a district general hospital. J Hosp Infect. 2003;54:104-8.

21. Landman D, Chockalingam M, Quale JM. Reduction in the incidence of methicillin-resistant Staphylococcus aureus and ceftazidime-resistant Klebsiella pneumoniae following changes in a hospital antibiotic formulary. Clin Infect Dis. 1999;28:1062-6.
22. Lautenbach E, LaRosa LA, Marr AM, Nachamkin I, Bilker WB, Fishman NO. Changes in the prevalence of vancomycin-resistant enterococci in response to antimicrobial formulary interventions: impact of progressive restrictions on use of vancomycin and thirdgeneration cephalosporins. Clin Infect Dis. 2003;36:440-6.

23. Meyer KS, Urban C, Eagan JA, Berger BJ, Rahal JJ. Nosocomial outbreak of Klebsiella infection resistant to late-generation cephalosporins. Ann Intern Med. 1993;119:353-8.

24. Pear SM, Williamson TH, Bettin KM, Gerding DN, Galgiani JN. Decrease in nosocomial Clostridium difficile-associated diarrhea by restricting clindamycin use. Ann Intern Med. 1994;120:272-7.

25. Leverstein-van Hall MA, Fluit AC, Blok HE, Box AT, Peters ED, Weersink AJ, et al. Control of nosocomial multiresistant Enterobacteriaceae using a temporary restrictive antibiotic agent policy. Eur J Clin Microbiol Infect Dis. 2001;20:785-91.

26. McNulty C, Logan M, Donald IP, Ennis D, Taylor D, Baldwin RN, et al. Successful control of Clostridium difficile infection in an elderly care unit through use of a restrictive antibiotic policy. J Antimicrob Chemother. 1997;40:707-11.

27. Bradley SJ, Wilson ALT, Allen MC, Sher HA, Goldstone AH, Scott GM. The control of hyperendemic glycopeptide-resistant Enterococcus spp. on a haematology unit by changing antibiotic usage. J Antimicrob Chemother. 1999;43:261-6.

28. Calil R, Marba ST, von Nowakonski A, Tresoldi AT. Reduction in colonization and nosocomial infection by multiresistant bacteria in a neonatal unit after institution of educational measures and restriction in the use of cephalosporins. Am J Infect Control. 2001;29:133-8.

29. de Champs C, Franchineau P, Gourgand JM, Loriette Y, Gaulme J, Sirot J. Clinical and bacteriological survey after change in aminoglycoside treatment to control an epidemic of Enterobacter cloacae. J Hosp Infect. 1994;28:219-29.

30. de Man P, Verhoeven BAN, Verbrugh HA, Vos MC, van den Anker JN. An antibiotic policy to prevent emergence of resistant bacilli. Lancet. 2000;355:973-8.

31. Singh N, Rogers P, Atwood CW, Wagener MM, Yu VL. Short-course empiric antibiotic therapy for patients with pulmonary infiltrates in the intensive care unit. A proposed solution for indiscriminate antibiotic prescription. Am J Respir Crit Care Med. 2000;162:505-11.

32. Toltzis P, Dul MJ, Hoyen C, Salvator A, Walsh M, Zetts L, et al. The effect of antibiotic rotation on colonization with antibiotic-resistant bacilli in a neonatal intensive care unit. Pediatrics. 2002;110:707-11.

33. Schein M, Assalia A, Bachus H. Minimal antibiotic therapy after emergency abdominal surgery. A prospective study. Br J Surg. 1994;81:989-91.

34. Jaskiewicz JA, McCarthy CA, Richardson AC, White KC, Fisher DJ, Dagan R, et al. Febrile infants at low risk for serious bacterial infection: an appraisal of the Rochester criteria and implications for management. Febrile Infant Collaborative Study Group. Pediatrics. 1994;94:390-6.

35. Leibovici L, Gitelman V, Yehezkelli Y, Poznanski O, Milo G, Paul M, et al. Improving empirical antibiotic treatment: prospective, nonintervention testing of a decision support system. J Intern Med. 1997;242:395-400.

36. Levin BR, Perrot V, Walker N. Compensatory mutations, antibiotic resistance and the population genetics of adaptive evolution in bacteria. Genetics. 2000;154:985-97.

37. Levin BR, Lipsitch M, Perrot V, Schrag S, Antia R, Simonsen L, et al. The population genetics of antibiotic resistance. Clin Infect Dis. 1997;24(Suppl 1):S9-16.

Address for correspondence: Peter Davey, Health Informatics Centre, University of Dundee Medical School, Mackenzie Building, Kirsty Semple Way, Dundee DD2 4BF, United Kingdom; fax: 44-1382-420-010; email: p.g.davey@chs.dundee.ac.uk 